\title{
Médicos y médicas, estilos asistenciales distintos: ¿satisfacción diferente de los usuarios?
}

\author{
Lourdes Biedma-Velázquez, Lic Cien Polit y Soc, (I) Rafael Serrano-del-Rosal, Dr Cien Polit y Soc.(I)
}

\author{
Biedma-Velázquez L, Serrano-del-Rosal R. \\ Médicos y médicas, estilos asistenciales \\ distintos: ¿satisfacción diferente de los usuarios? \\ Salud Publica Mex 2009;5 I:277-284.
}

\section{Resumen}

Objetivo.Verificar la existencia de diferencias en la satisfacción de los usuarios de los servicios sanitarios, según sea que la atención se reciba de un médico hombre o mujer. Material y métodos. Se utilizó la encuesta realizada a usuarios de atención primaria en 2005 que llevó a cabo el Instituto de Estudios Sociales Avanzados, del Consejo Superior de Investigaciones Científicas (IESA/CSIC). Se efectuó un análisis bivariado entre el sexo del médico y las variables de satisfacción, y un análisis de varianza (ANOVA); se tomó como variable dependiente el indicador de satisfacción general y como variables independientes las características del individuo y del sistema, entre estas últimas el sexo del médico. Resultados. En el análisis bivariado se constató la relación entre sexo del médico y la satisfacción con algunos de los elementos del servicio, si bien en el análisis de dependencia, que incluye variables sociodemográficas y del sistema, esta influencia desaparece. Conclusión. Se confirmaron las diferencias en la práctica asistencial de hombres y mujeres médicos, pero no así las diferencias en la satisfacción general con el servicio recibido.

Palabras clave: satisfacción del paciente; salud; género; atención primaria de salud; asistencia médica
Biedma-Velázquez L, Serrano-del-Rosal R.

Male physicians and female physicians,

different medical styles: different users' satisfaction?

Salud Publica Mex 2009;5 I:277-284.

\section{Abstract}

Objective. To analyse differences in the satisfaction of health service users associated with the sex of the attending doctor. Material and Methods. Data obtained from the Primary Care Services User Survey (2005), part of a project regarding user satisfaction with the Andalusian Public Health Services. A bivariate analysis was conducted, the two variables being doctors' sex and user satisfaction, as was an ANOVA, taking as a dependent variable the indicator of general satisfaction and as independent variables the characteristics of the individual and that of the system, including physician sex. Results. In the bivariate analysis a relation was confirmed between doctors' sex and satisfaction with the components of the health service received. Nevertheless, this influence disappears in the analysis of dependence, which includes sociodemographic and system variables. Conclusion. Differences between practices by male and female doctors are confirmed, but not the differences in general satisfaction with the service received.

Key words: patient satisfaction; health;gender; primary health care; medical assistance

Fecha de recibido: 6 de mayo de 2008 - Fecha de aprobado: 30 de marzo de 2009 Solicitud de sobretiros: Dr. Rafael Serrano del Rosal. Instituto de Estudios Sociales Avanzados de Andalucía. Consejo Superior de Investigaciones Científicas. Calle Campo Santo de los Mártires, 7. I4004-Córdoba, España.

Correo electrónico: rserrano@iesa.csic.es 
U n elemento clave para la política sanitaria en las sociedades occidentales contemporáneas es la mejoría de la calidad de los servicios suministrados a los ciudadanos. ${ }^{1}$ Bajo este enfoque, el análisis de la satisfacción del usuario es de gran importancia para el conocimiento y gestión de tales servicios, en particular por la estrecha y confirmada relación existente entre la satisfacción y la calidad de las prestaciones ofrecidas. ${ }^{2}$ Este enfoque pone el acento en las necesidades y expectativas del usuario y considera a éste "juez" de la organización sanitaria, ${ }^{3-5}$ con capacidad de mostrar al gestor elementos e información que no le es posible obtener por otros medios. Además, desde el punto de vista estrictamente médico y de salud pública, se advierte que un paciente satisfecho se muestra más predispuesto a seguir las recomendaciones médicas y terapéuticas y, en consecuencia, a mejorar su salud..$^{6-9}$

La percepción sobre la calidad de los servicios sanitarios depende en grado sustancial tanto del resultado final del tratamiento o la atención prestada como del proceso completo por el cual se lleva a cabo la prestación sanitaria, con especial atención, como ya lo señaló Donabedian, ${ }^{10,11}$ a la relación médico-paciente que contribuirá enormemente a su percepción y valoración.

En consecuencia, la satisfacción del paciente es un concepto complejo de naturaleza multidimensional. ${ }^{12,13}$ Estas dimensiones se pueden medir y evaluar de manera separada, ${ }^{14}$ tras analizar la importancia relativa que cada individuo concede a cada una de ellas. ${ }^{13,15}$

Si todo ello no tuviera una complejidad suficiente, se debe tener en cuenta también que la satisfacción del usuario está influida por las propias características sociodemográficas y socioeconómicas del individuo, que si bien no condicionan dicha satisfacción, sí la matizan. Así, se ha contrastado por numerosos estudios, ${ }^{16-18}$ incluida la propia experiencia de este grupo de investigación. ${ }^{19-21}$

Por último, junto a las características de la demanda (de los usuarios), no deben soslayarse tampoco las diferencias que surgen de la oferta, esto es, de las propias condiciones o características en las que se efectúa la prestación del servicio. Ejemplo de esta clase de características puede ser el tipo de centro sanitario (centro de salud o consultorio local) o el sexo del prestador del servicio.

El enfoque de género en salud, entendido el género en su concepción más social, distinto de la categoría sexo, ${ }^{22}$ se ha centrado en las diferencias de salud y autopercepción de salud en mujeres y hombres. No obstante, en los últimos años han surgido estudios que tratan de analizar si existen diferencias en la práctica asistencial de hombres y mujeres médicos. ${ }^{23-25}$

La incorporación masiva de las mujeres al mundo laboral, y en concreto su notable presencia en el ámbito sanitario, cada vez más como médicos, pone de relieve la concurrencia del género como variable necesaria para la interpretación de las diferencias en la práctica asistencial de estos profesionales. Las mujeres se han incorporado a la profesión médica, pero no lo han hecho de forma igualitaria en todos los ámbitos o especialidades; además, dicha incorporación ha estado cargada de valores y/o desigualdades de género..$^{25,26}$

Parece evidente que los cambios sociales y la incorporación de la mujer a la profesión médica han producido algún efecto en la práctica profesional. Son numerosos los estudios que han tratado de analizar dicho impacto y señalan, por lo general, que las médicas mantienen de forma habitual consultas más largas, otorgan mayor importancia a la comunicación médicopaciente sobre la base de aspectos interpersonales y emocionales, suministran mayor información y, en general, mantienen una relación más asertiva. ${ }^{23,25,26} \mathrm{No}$ obstante, la explicación empírica acerca de la existencia de diferencias en la satisfacción del paciente, según el género del médico, es escasa y contradictoria. ${ }^{24}$

El objetivo principal de este trabajo es conocer si la satisfacción de los usuarios con los servicios sanitarios que reciben, en este caso en Andalucía, son diferentes de acuerdo con el género del prestador del servicio, con base en sus diferentes prácticas asistenciales.

\section{Material y métodos}

Se han utilizado los datos procedentes del estudio Opinión y Satisfacción de los Usuarios con los Servicios de Atención Primaria del Servicio Andaluz de Salud (SAS) del año 2005 (E-0505), un protocolo realizado por el Instituto de Estudios Sociales Avanzados, del Consejo Superior de Investigaciones Científicas (IESA-CSIC), mediante convenio de colaboración con el SAS/Junta de Andalucía. * En este estudio se realizó un total de 22594 encuestas en 552 consultorios locales, 92 consultorios auxiliares y 369 centros de salud. De ellas, en 18

\footnotetext{
* Este estudio forma parte del Plan Estadístico de Andalucía 20032006, Ley 8/ 2002 del 17 de diciembre (ley que regula la producción de estadísticas oficiales en el periodo 2003-2006, en el marco de la Comunidad Autónoma Andaluza), por lo que ha sido aprobado por el Gobierno de Andalucía y ha pasado todos los requerimientos metodológicos, éticos y legales que de ello se derivan. Además, este proyecto de investigación se ha sometido a los condicionamientos que adicionalmente imponen el Comité de Ética y Buenas Prácticas y el Comité Científico Técnico para la Calidad de la Investigación del IESA-CSIC. Los usuarios que contestaron a las encuestas eran en todos los casos mayores de edad y lo hicieron libremente una vez informados de quién realizaba las encuestas, del objetivo general de las mismas y de garantizarles legalmente la confidencialidad de sus opiniones y datos personales según la ley 12/89 que regula el secreto estadístico para las administraciones públicas.
} 
894 casos (83.62\%) había una respuesta efectiva sobre el sexo del médico de cabecera o pediatra. Las entrevistas se realizaron de forma presencial, con selección aleatoria de los individuos a la salida de los centros, una vez finalizada la visita médica. El muestreo se estratificó por conglomerados y cada conglomerado fue un consultorio o centro de salud, con submuestreo aleatorio sistemático de personas dentro de cada conglomerado y cobertura de cuotas de sexo y edad, según fuera el número de visitas diarias a cada centro. El diseño consistió en tantas muestras independientes como distritos sanitarios, con al menos 400 entrevistas en cada distrito, distribuidas de manera proporcional de acuerdo con el tipo de centro, tamaño del municipio, grupos de edad y sexo de los usuarios. Dicha muestra tiene, para el caso de Andalucía, un error máximo a priori de $\pm 1 \%$ para un nivel de confianza de dos sigmas. Los datos se procesaron con el paquete estadístico SPSS, 12.0 y 15.0, mediante su módulo de Muestras Complejas, que incluye la ponderación de las distintas unidades de análisis que componen el diseño muestral, de acuerdo con su peso en la población estudiada.

El diseño metodológico de la investigación que sustenta este artículo se estructura y cobra sentido en las siguientes fases:

1. En una primera fase se realizó un análisis bivariado entre el sexo del médico y las diferentes variables de satisfacción de los usuarios. Para ello se ha realizado un análisis de asociación de variables a través de los estadísticos ji cuadrada de Pearson y V de Cramer, entre la variable sexo del médico o pediatra y los diferentes indicadores de satisfacción del cuestionario. Las respuestas se presentan a través de indicadores, formados por el porcentaje de usuarios que han señalado estar satisfechos ("muy satisfecho" y "satisfecho" o "muy bueno" y "bueno") e insatisfechos ("muy insatisfecho", "insatisfecho" y "ni satisfecho ni insatisfecho", o "muy malo", "malo" y "ni bueno ni malo") con cada uno de los aspectos del servicio recibido. Se excluyó a todas las personas que contestaron "no sabe" o "no contesta", y en su caso "no procede", y se obtuvo un indicador con valor 0 si no estaba satisfecho y 1 si lo estaba.

Debido al posible efecto de la desigual distribución de los médicos de ambos sexos por centros de salud o consultorios, se realizó también un análisis de residuos y se examinaron de forma combinada las características de la oferta y la demanda; el análisis se centró de manera especial en observar si el perfil de los usuarios de médicos o pediatras hombres era diferente al de los usuarios de médicas o pediatras mujeres.

El último paso dentro de esta primera fase fue la construcción de una variable de satisfacción global robusta que sirviera como variable dependiente en posteriores análisis, y a la que se denominó satisfacción general. El proceso de creación del indicador resumen ha seguido un procedimiento prácticamente idéntico al desarrollado en el artículo " $\mathrm{La}$ anatomía de la satisfacción del paciente" publicado en esta revista. ${ }^{20,21}$ En consecuencia, se resumieron las tres variables de satisfacción del cuestionario a través de un análisis de componentes principales categórico (CATPCA).

Una vez obtenida la variable satisfacción general, se realizó una prueba de comparación de medias para conocer si existían diferencias significativas en la satisfacción general de los usuarios, según fuera el sexo del médico que les atendió.

2. En una segunda fase se efectuó un análisis de dependencia (ANOVA) y se tomó como variable dependiente la satisfacción general y como variables independientes las características sociodemográficas del individuo y las características del sistema, entre estas últimas el sexo del prestador del servicio. La confluencia de diferentes variables con acción sinérgica en la satisfacción de los usuarios podía dificultar la correcta interpretación de los resultados del análisis bivariado; por esa razón se realizó este análisis multivariado que da cuenta del efecto de cada variable sobre la variable dependiente, de tal forma que se dejaron ceteris paribus los efectos de las demás.

\section{Resultados}

\section{Primera fase: análisis bivariado}

Las variables que se muestran en el cuadro I resultaron significativamente distintas de acuerdo con el sexo del médico/ pediatra. También se muestran en dicho cuadro los resultados desagregados, de acuerdo con el sexo del entrevistado. Por consiguiente, en general, son las mujeres usuarias las que presentan diferencias más acusadas en sus valoraciones según se trate del sexo del médico / pediatra que las atendió; este hecho es importante en el caso de los usuarios tan sólo en dos indicadores: el tiempo que les dedica su médico y la facilidad para identificar a los profesionales.

Por otro lado, en general, los/as médicos/ as se valoran de igual forma por el conjunto de usuarios del SAS, 
pero no así si se tiene en cuenta tan sólo a las usuarias, entre las que se observa una mejor valoración de los médicos de su mismo sexo. No debe soslayarse esto, ya que los principales usuarios del sistema sanitario son mujeres, no sólo por su mayor longevidad, sino también porque a su papel de pacientes hay que añadir el de cuidadoras.

En general, los usuarios se muestran más satisfechos con el centro, la forma en que resolvieron los trámites administrativos, el tiempo de espera, la organización del centro en general, la disposición del personal para escucharlos, además de que recomendaron más el centro cuando su médico fue un hombre. Lo contrario se observa en la valoración de la facilidad para identificar a los profesionales, y el tiempo que les concede su médico, un área en la cual los usuarios juzgan mejor a su médico cuando es mujer.

Los aspectos que presentan mayor diferenciación son los relacionados con el tiempo de espera hasta entrar en la consulta y la satisfacción con el tiempo que les dedica el profesional médico. En efecto, los usuarios están más insatisfechos con el tiempo que deben esperar cuando el médico/pediatra es una mujer $\mathrm{y}$, tal como aparece en otros estudios, están más satisfechos con el tiempo que les dedican en la consulta estas profesionales. ${ }^{27,28}$

En cuanto a las principales preguntas de satisfacción, se observa una relación entre la satisfacción general con el servicio (p2) y la recomendación a un familiar o amigo (p54), pero hay que destacar la inexistencia de diferencias en la confianza en la asistencia de acuerdo con el género del prestador del servicio (p20). Según los estudios anteriores, ${ }^{21}$ estas tres variables miden de forma sustancial lo mismo y pueden resumirse en un indicador más general y completo, y al que se ha denominado satisfacción general, que resume $56.83 \%$ (autovalor del 1.1705) de la información contenida en las tres preguntas anteriores. Debido a la naturaleza métrica de esta nueva variable, se ha realizado un análisis de asociación con el sexo del médico a través de la prueba $\mathrm{F}$, que permite concluir que

Cuadro I

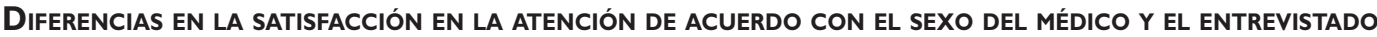

\begin{tabular}{|c|c|c|c|c|c|c|c|c|c|c|}
\hline & \multirow{2}{*}{ \% Satisfacción según el género del médico } & \multicolumn{3}{|c|}{ Entrevistados hombres } & \multicolumn{3}{|c|}{ Entrevistadas mujeres } & \multicolumn{3}{|c|}{ Total entrevistados } \\
\hline & & $\begin{array}{l}\text { \% Médico } \\
\text { hombre/ } \\
\text { mujer }\end{array}$ & Dif. & $\begin{array}{l}V \text { Cramer } \\
\text { y sig }\end{array}$ & $\begin{array}{l}\text { \% Médico } \\
\text { hombre/ } \\
\text { mujer }\end{array}$ & Dif. & $\begin{array}{l}V \text { Cramer } \\
\text { y sig }\end{array}$ & $\begin{array}{l}\text { \% Médico } \\
\text { hombrel } \\
\text { mujer }\end{array}$ & Dif. & $\begin{array}{l}\text { V Cramer } \\
\text { y sig }\end{array}$ \\
\hline P2 & Satisfacción con el centro & $87.2-86.4$ & 0.8 & ns & $86.4-84.9$ & 1.5 & $0.021^{*}$ & $86.7-85.3$ & 1.4 & $0.019^{\ddagger}$ \\
\hline P5 & Facilidad en trámites y papeles & $74.7-74.2$ & 0.5 & ns & $73.6-69.9$ & 3.7 & $0.04 I^{\ddagger}$ & $74.0-71.1$ & 2.9 & $0.032^{\ddagger}$ \\
\hline P6 & Tiempo de espera & $48.1-43.3$ & 4.8 & $0.047^{\ddagger}$ & $48.5-41.0$ & 7.5 & $0.074^{\ddagger}$ & $48.4-4 I .7$ & 6.7 & $0.066^{\ddagger}$ \\
\hline PII & Identificación de los profesionales & $83.1-87.1$ & -4.0 & $0.052^{\ddagger}$ & $83.1-85.2$ & -2.1 & $0.029 \ddagger$ & $83.1-85.7$ & -2.6 & $0.035^{\ddagger}$ \\
\hline $\mathrm{PI} 2$ & Tiempo de dedicación del médico & $90.7-92.3$ & -1.6 & $0.027^{*}$ & $89.8-91.3$ & -1.5 & $0.025^{\ddagger}$ & $90.1-91.6$ & -1.5 & $0.025^{\ddagger}$ \\
\hline $\mathrm{P} 20$ & Confianza en la asistencia recibida & $85.7-85.0$ & 0.7 & ns & $85.0-84.9$ & 0.1 & ns & $85.3-84.9$ & 0.4 & ns \\
\hline P26 & Organización del centro en general & $77.0-75.8$ & 1.2 & ns & 75.9-73.0 & 2.9 & $0.033^{\ddagger}$ & $76.3-73.8$ & 2.5 & $0.029 \ddagger$ \\
\hline P29 & Disponibilidad del personal para escucharlo & $85.1-86.1$ & -1.0 & ns & $83.4-81.3$ & 2.1 & $0.027^{\ddagger}$ & $84.0-82.7$ & 1.3 & $0.018^{*}$ \\
\hline P30x & $\begin{array}{l}\text { Valoración de los médicos de cabeceral } \\
\text { pediatras }\end{array}$ & $95.2-95.9$ & -0.7 & ns & $93.2-94.2$ & -1.0 & $0.021^{*}$ & $93.9-94.7$ & -0.8 & $0.017^{*}$ \\
\hline P54 & Recomendaría el centro & $89.2-88.9$ & 0.3 & ns & $89.4-87.2$ & 2.2 & $0.034^{\ddagger}$ & $89.3-87.7$ & 1.6 & $0.026^{\ddagger}$ \\
\hline \multicolumn{11}{|c|}{ * Indica que en esa muestra las diferencias en salud subjetiva alcanzan niveles estadísticamente significativos con una $p<0.05$} \\
\hline \multicolumn{11}{|c|}{$\begin{array}{l}\text { Dif. = diferencia en el porcentaje de satisfacción de las personas atendidas por un médico hombre y las atendidas por un médico mujer } \\
n s=\text { indica que las diferencias no son significativas al } 95 \%\end{array}$} \\
\hline
\end{tabular}


existen diferencias en las medias de satisfacción entre los usuarios que han acudido a un doctor o una doctora.

Como último paso de esta primera fase se ha tratado de determinar si en las valoraciones que realizan los entrevistados pueden influir las características de la oferta y la demanda del servicio, es decir, que los usuarios no se distribuyan de igual forma entre médicos y médicas, o que las características propias del servicio no sean iguales en las consultas de unos y otras. Se realizó un análisis de residuos tras dividir la población estudiada según fuera el sexo del médico al que acudió. Hay que destacar, en este sentido, que los usuarios no se distribuyen de forma homogénea entre médicos y médicas, lo cual es un hecho bastante significativo, sobre todo al considerar la libre elección del profesional que existe en la comunidad autónoma andaluza. En general, para el caso de los médicos de cabecera, han recibido mayor atención más personas por un hombre que por una mujer; en cambio, en el caso de la pediatría, hay más usuarios atendidos por pediatras mujeres que por hombres (cuadro II). Por tipo de centro, se observó un mayor número de mujeres médico en centros de salud frente a los consultorios y, en estrecha relación con este indicador, un mayor porcentaje de usuarios que acuden a ellas en poblaciones mayores de 100000 habitantes. Los hombres reciben atención en proporción mayoritaria por médicos de su mismo sexo, un fenómeno observado en menor medida en el caso de las mujeres. También se advierte un mayor porcentaje de personas con médico de cabecera/pediatra mujer en el grupo de edad más joven respecto de los mayores de 65.

\section{Segunda fase: ANOVA}

Se ha realizado un análisis multivariado en el que, tal y como puede observarse en el cuadro III, la variable dependiente, satisfacción general, está explicada en $4.3 \%$ por las características sociodemográficas del encuestado

Cuadro II

Principales variables sociodemográficas y de sistema de acuerdo con el seXo del médico o el Pediatra

\begin{tabular}{|c|c|c|c|c|c|c|}
\hline \multirow{5}{*}{ Sexo del entrevistado } & \multicolumn{6}{|c|}{ Sexo del médico o el pediatra } \\
\hline & \multirow[b]{3}{*}{ Hombre } & Hombre & Mujer & Hombre & Mujer & \multirow{3}{*}{ Sig } \\
\hline & & \multicolumn{2}{|c|}{$\%$ columnas } & \multicolumn{2}{|c|}{$\%$ filas } & \\
\hline & & $35.8 \%$ & $28.6 \%$ & $65.1 \%$ & $34.9 \%$ & \\
\hline & Mujer & $64.2 \%$ & $71.4 \%$ & $57.3 \%$ & $42.7 \%$ & 0.000 \\
\hline \multirow{4}{*}{ Edad del entrevistado } & 29 años o menos & $14.2 \%$ & $14.9 \%$ & $58.8 \%$ & $41.2 \%$ & \multirow{4}{*}{0.000} \\
\hline & 30 a 45 años & $26.8 \%$ & $31.7 \%$ & $55.7 \%$ & $44.3 \%$ & \\
\hline & 46 a 65 años & $29.6 \%$ & $28.1 \%$ & $61.1 \%$ & $38.9 \%$ & \\
\hline & 66 años o más & $29.4 \%$ & $25.3 \%$ & $63.4 \%$ & $36.6 \%$ & \\
\hline \multirow{3}{*}{ Tipo de centro } & Centro de salud & $77.9 \%$ & $83.1 \%$ & $58.3 \%$ & $41.7 \%$ & \multirow{3}{*}{0.000} \\
\hline & Consultorio local & $20.7 \%$ & $15.6 \%$ & $66.4 \%$ & $33.6 \%$ & \\
\hline & Consultorio auxiliar & $1.5 \%$ & $1.3 \%$ & $62.4 \%$ & $37.6 \%$ & \\
\hline \multirow{7}{*}{ Nivel educativo } & Analfabetos & $6.8 \%$ & $5.1 \%$ & $66.7 \%$ & $33.3 \%$ & \multirow{7}{*}{0.000} \\
\hline & Estudios primarios sin completar & $44.2 \%$ & $40.5 \%$ & $61.9 \%$ & $38.1 \%$ & \\
\hline & Primarios completos, ESO, EGB & $24.9 \%$ & $26.3 \%$ & $58.5 \%$ & $41.5 \%$ & \\
\hline & FP I, ciclos formativos de grado medio & $4.9 \%$ & $5.6 \%$ & $57.0 \%$ & $43.0 \%$ & \\
\hline & Bachillerato, BUP, COU, ciclos sup., FP II & $11.7 \%$ & $13.2 \%$ & $56.8 \%$ & $43.2 \%$ & \\
\hline & Universitarios de grado medio & $4.9 \%$ & $6.5 \%$ & $53.1 \%$ & $46.9 \%$ & \\
\hline & Universitarios de grado superior o $3^{\text {er }}$ ciclo & $2.5 \%$ & $2.9 \%$ & $59.8 \%$ & $40.2 \%$ & \\
\hline \multirow{2}{*}{ Tipo de visita } & Médico de cabecera & $87.7 \%$ & $78.2 \%$ & $62.6 \%$ & $37.4 \%$ & \multirow{2}{*}{0.000} \\
\hline & Pediatra & $11.1 \%$ & $20.6 \%$ & $44.7 \%$ & $55.3 \%$ & \\
\hline \multirow{3}{*}{ Tamaño del hábitat } & Menos de $5000 \mathrm{hb}$ & $19.8 \%$ & $15.2 \%$ & $66 \%$ & $34 \%$ & \multirow{3}{*}{0.000} \\
\hline & 5000 a $100000 \mathrm{hb}$ & $48.4 \%$ & $44 \%$ & $62.1 \%$ & $37.9 \%$ & \\
\hline & Más de $100000 \mathrm{hb}$ & $31.8 \%$ & $40.8 \%$ & $53.7 \%$ & $46.3 \%$ & \\
\hline
\end{tabular}

ESO= educación secundaria obligatoria, $\mathrm{EGB}=$ educación general básica, $\mathrm{FP} \mathrm{I}=$ formación profesional de primer grado, $\mathrm{BUP}=$ bachillerato unificado polivalente, $\mathrm{COU}=$ curso de orientación universitario, FP $\|=$ formación profesional de segundo grado

Fuente: Comunidad Autónoma de Andalucía (España), 2005. Estudio E0505 (Instituto de Estudios Sociales Avanzados, del Consejo Superior de Investigaciones Científicas) 


\section{Cuadro III}

Resumen del MOdelo

\begin{tabular}{|c|c|c|c|c|c|}
\hline & \multicolumn{3}{|c|}{$\begin{array}{l}\text { Modelo general lineal univariante (ANOVA). } \\
\text { Prueba de medias.Variable dependiente: satisfacción general }\end{array}$} & \multirow[b]{2}{*}{ Potencia } & \multirow[b]{2}{*}{ Importancia de Pratt } \\
\hline & $F$ & Sig & Eta cuadrado parcial & & \\
\hline Modelo corregido & 19.314 & 0.000 & 0.045 & 1.00 & \\
\hline Constante & 35193.294 & 0.000 & 0.683 & 1.00 & \\
\hline Sexo del entrevistado & 0.019 & 0.891 & 0.000 & 0.05 & \\
\hline Grupos de edad & 38.274 & 0.000 & 0.007 & 1.00 & $32.3 \%$ \\
\hline Estado civil & 2.385 & 0.049 & 0.001 & 0.69 & $2.7 \%$ \\
\hline Nivel educativo & 3.464 & 0.002 & 0.001 & 0.95 & $5.9 \%$ \\
\hline Situación de convivencia & 4.453 & 0.000 & 0.002 & 0.99 & $10.1 \%$ \\
\hline Ocupación & 1.165 & 0.316 & 0.001 & 0.55 & $2.6 \%$ \\
\hline Tipo de centro & 19.837 & 0.000 & 0.002 & 1.00 & $11.2 \%$ \\
\hline Tipo de visita & 21.814 & 0.000 & 0.001 & 0.99 & $6.2 \%$ \\
\hline Tipo de cita & 18.449 & 0.000 & 0.002 & 1.00 & $10.4 \%$ \\
\hline Tamaño del hábitat & 24.970 & 0.000 & 0.003 & 1.00 & $14.1 \%$ \\
\hline Frecuentación & 6.558 & 0.001 & 0.001 & 0.91 & $3.7 \%$ \\
\hline Sexo del médico/pediatra & 3.149 & 0.076 & 0.000 & 0.43 & \\
\hline
\end{tabular}

Valor de contraste alfa $=0.05$

$R$ cuadrado $=0.045$ ( $R$ cuadrado ajustado $=0.043)$

Fuente: Comunidad Autónoma de Andalucía (España), 2005. Estudio E0505 (Instituto de Estudios Sociales Avanzados, del Consejo Superior de Investigaciones Científicas)

Nota aclaratoria (I): se ha utilizado la importancia de Pratt, que indica qué variable tiene mayor influencia en la variable dependiente. Este estadístico resulta de multiplicar el coeficiente de regresión de cada variable por la correlación con la variable dependiente y dividir el resultado por el R2 del modelo. La suma de todas las importancias es 100

Nota aclaratoria (2): debe recordarse que el análisis de la varianza debe cumplir con una serie de supuestos. El primero de ellos, el de normalidad de la variable dependiente, en este caso, según la prueba de Kolmogorov-Simirnov, no se verifica. Sin embargo, la no normalidad no afecta de forma decisiva a las conclusiones, por la aplicación del teorema central del límite (si se tiene un grupo numeroso de variables independientes y todas ellas siguen el mismo modelo de distribución, cualquiera que sea éste, la suma de ellas se distribuye según una distribución normal). Otra hipótesis que se ha de cumplir es la igualdad de varianzas en todos los grupos y, dado que éstos son aproximadamente del mismo tamaño, no es necesario verificar. Por último, la independencia de las muestras que conforman cada grupo está asegurada debido a la forma en que se obtuvieron los datos, mediante muestreo aleatorio

y las características del sistema. No es un porcentaje muy elevado, ya que no puede esperarse que la satisfacción dependa de este tipo de características, pero tiene importancia suficiente para analizar de manera detenida la forma en que afectan.

La edad es, sin duda alguna, la variable externa al sistema sanitario que más influye en la satisfacción del paciente. El nivel de estudios modifica sobre todo los tramos más altos, de forma tal que los universitarios y los que tienen estudios medios están más insatisfechos. En cuanto a la ocupación, los jubilados, y otras personas que se dedican a las labores del hogar, son los más satisfechos. El estado civil afecta también a la satisfacción global y son los individuos casados o los que conviven en pareja los más críticos, en comparación con los solteros, separados/divorciados o viudos. En cuanto a la situación de convivencia, se observa que las familias nucleares y monoparentales son en términos generales las más insatisfechas.

También influyen en la satisfacción variables como el tipo de centro: los consultorios se valoran de forma más positiva que los centros de salud. Muy relacionado con este indicador se encuentra el tamaño de hábitat, ya que los centros no se distribuyen de igual forma en todos los núcleos poblacionales. Las personas que viven en municipios de menos de 5000 habitantes son los más satisfechos y por debajo se hallan los que viven en municipios "medios" de 5000 a 100000 habitantes. En cuanto al tipo de cita, están más satisfechos los individuos que han acudido con cita previa, ya sea solicitada por teléfono o concertada en el centro, en comparación con las personas que reciben atención sin cita ni número. 
La frecuentación incide también en la satisfacción y son más positivas las valoraciones cuanto más se acude al centro sanitario. Por último, en cuanto al tipo de visita, debe señalarse que los sujetos que han acudido a un médico de cabecera están algo más satisfechos respecto de quienes acuden al pediatra. Esta diferencia introduce otra más sutil: la diferencia entre ser paciente y ser acompañante, en este caso de un menor.

A un nivel de confianza de $95 \%$, se observa que la variable que no afecta al comportamiento de la satisfacción general es el sexo, tanto del entrevistado como del médico / pediatra. El sexo del entrevistado no ejerce influencia alguna en la satisfacción del usuario con el servicio recibido. Asimismo, el sexo del médico o pediatra no modifica la satisfacción general del usuario, es decir, a pesar de que en el plano individual ciertas variables, tanto de satisfacción general como de algunos aspectos concretos del servicio, mostraban diferencias relacionadas con el sexo del prestatario médico, al controlar la concurrencia de otros factores que pueden actuar al mismo tiempo, a modo de variables "mediadoras", esta relación desaparece. No obstante, esta afirmación debe matizarse, ya que como se ha comprobado, si bien el sexo del médico no altera su satisfacción global, sí es muy importante analizar otros aspectos parciales concretos, como su satisfacción con el tiempo de espera o el tiempo que los atienden en la consulta.

\section{Discusión}

La información empírica apoya la existencia de diferencias en la práctica asistencial de médicos hombres y mujeres, ya que se atribuye por ejemplo a estas últimas el desarrollo de consultas más largas, lo cual se observa en un incremento de la satisfacción con el tiempo de consulta en los usuarios que reciben atención de ellas, aunque también en el hecho de una mayor insatisfacción de éstos con el tiempo de espera hasta entrar a la consulta. Estos hallazgos son coherentes con los identificados en las publicaciones sobre práctica asistencial, de acuerdo con el género del médico, aunque no siempre indican que los usuarios están, en general, más satisfechos con unas o con otros.

Se han encontrado también diferencias en la satisfacción de los usuarios, en relación con el sexo del médico, con aspectos organizativos del centro y las instalaciones; esto puede ser un efecto de las diferencias en la oferta, es decir, el tipo de centro, el ámbito poblacional, y otros más, en los que mayoritariamente prestan sus servicios unos y otros, o también de las características de la demanda, es decir, que sea la distribución desigual de los usuarios entre médicos de diferente sexo la fuente de una diferente valoración del entorno organizativo y las consultas de estos profesionales. En este sentido, se ha demostrado que los usuarios no se distribuyen de forma homogénea entre médicos de ambos sexos.

Por otra parte, es también destacable que el género del médico no influye en algo tan fundamental como la confianza en la asistencia sanitaria, o en la valoración de médicos y pediatras, lo cual sugiere que las diferencias reconocidas en la satisfacción general -según el sexo- se basan en elementos distintos de la profesionalidad y competencia de los médicos/as. En términos generales, los usuarios tienen los mismos niveles de confianza en médicos hombres y mujeres, pero no se sienten igual de satisfechos con otros aspectos.

En consecuencia, si bien no existe una relación clara entre el sexo del médico y la satisfacción general del paciente, sí ocurre dicha relación entre ser mujer u hombre médico y la satisfacción con diferentes elementos del sistema. Es probable que esto haya llevado a algunos autores a considerar sin más comprobación empírica que existe una relación clara entre sexo del médico y satisfacción general con el servicio sanitario. No obstante, cuando se analiza este hecho de forma detallada se encuentra que, en términos agregados, los usuarios valoran de manera equivalente a hombres y mujeres médicos, pero diferencian aspectos en su práctica asistencial, como el tiempo concedido por su médico, el tiempo que deben aguardar desde que llegan a la consulta hasta que se los atiende, la identificación de los profesionales, etcétera.

Por lo tanto, es el género, entendido como constructo social que trasciende al sexo, o más bien las desigualdades de género, lo que podría hallarse en la base de la inequitativa distribución de hombres y mujeres médicos en la estructura sanitaria o la desigual elección de profesionales de los pacientes; todo ello, junto con una práctica asistencial diferenciada, afecta las diferencias en la satisfacción del usuario según sea el sexo del médico. No obstante, a pesar de que hombres y mujeres médico mantengan estilos profesionales distintos, no se producen diferencias significativas en la satisfacción de los usuarios, salvo en aspectos muy concretos o en elementos en los que puede influir la distinta distribución de las médicas dentro de la profesión y no en su satisfacción general con la asistencia o la confianza que depositan en los profesionales que los atienden. Es interesante tratar de responder por qué existe una distribución diferente de mujeres y hombres médicos en el mapa sanitario del SAS; aunque esto se constata en este trabajo, no es objeto de su estudio ni se dispone de elementos suficientes para explicarlo, pero en futuros análisis se intentará contrastar la hipótesis de que es la compatibilidad de su profesión con otras asignaciones de género, y su tardía incorporación a la profesión médica y al desarrollo de su profesión en el sistema sanitario 
analizado, lo que puede hallarse en la base de dicho fenómeno, como ya apuntan diferentes autoras. ${ }^{29}$

\section{Agradecimientos}

Los autores agradecen al SAS su motivación y colaboración y al Ministerio de Educación y Ciencia, Plan Nacional I+D+I (SEJ2005-06099) el apoyo financiero. Asimismo, de manera especial, están en deuda con Julia Ranchal, José M ${ }^{a}$ García, Nuria Loriente y otros colegas de la UTEA del IESA.

\section{Referencias}

I. Ruiz M, Martínez G, Calvo J, Aguirre H,Arango R, Lara R, et al. Bases para la evaluación de la calidad de la atención en las unidades médicas del sector salud. Salud Publica Mex 1990;32:156-169.

2. Steiber R. Measuring and manaing patient satisfaction. J Am Hosp Publ 1988:1-23.

3. Parasuman A, ZeilthamlV, Berry L.A conceptual model of service quality and its implications for future research.J Mark 1985;49(4):41-50.

4. Parasuman A, ZeilthamlV, Berry L.Alternative scales for measuring service quality: a comparative assessment based on psychometric and diagnostic criteria.J Mark 1994;70(3):20I-230.

5. Donabedian A. Continuidad y cambio en la búsqueda de la calidad. Salud Publica Mex 1993;35:238-247.

6. Mira JJ,Vitaller J,Aranaz JM, Herrero JF, Buill FA. La satisfacción del paciente. Concepto y aspectos metodológicos. Rev Psicol Sal 1992;4:89-II6. 7. Carminal J. La medida de la satisfacción: un instrumento de participación de la población en la mejora de la calidad de los servicios sanitarios. Rev Cal Asis 200I; I6(4):276-279.

8. Linder-Pelz S. Toward a theory of patient satisfaction. Soc Sci Med 1982;16(5):577-582.

9.Williams B. Patient satisfaction: a valid concept? Soc Sci Med 1994;38(4):509-516.

10. Donabedian A. La calidad de la atención médica. Definición y métodos de evaluación. México: Copilco, 1984.

II. Donabedian A. Evaluating the quality of medical care. Milbank Quart 2005;83(4):69I-729.

12. Steiber SR, KrowinskiWJ. Mearuring and managing patient satisfaction. Ame Hosp Publ 1996;1-23.
13. Mead N, Bower P, Hann M. The impact of general practitioners "patient-centredness on patients" post-consultation satisfaction and enablement. Soc Sci Med 2002;55(2):283-299.

14. Fitzpatrick R. Surveys of patient satisfaction: II- Designing a question and onducting a survey. Br Med J 1991;302: I I29-I I 32.

15.Ware JE, Snyder MK, Wricht WR, Davies AR. Defining and Measuring patient satisfaction with medical care. Eval Prog Plan 1983;6:247-263. 16. Delgado A, Martínez-Cañavate T, García V, Frías J, Rueda T, Morata J. Preferencias y estereotipos de los pacientes sobre el género del médico de familia. Rev Aten Prim 1999;23(5):268-274.

17. García-Arqué RM. Actitudes de la población ante el sector sanitario. Colección opiniones y actitudes. CIS 1997; 17.

18. Esteban-de la Rosa MA. Satisfacción de los usuarios: factores condicionantes. Rev ROL Enfer 1994; 188:33-39.

19. Serrano R, Biedma L. El usuario del sistema sanitario: gestor de calidad. IX Congreso de Metodología de las Ciencias Sociales y de la Salud, Granada, Libro de resúmenes, 2005:262.

20. Serrano-del Rosal R, Loriente-Arín L. La anatomía de la satisfacción del paciente. Salud Publica Mex 2008(50): I-I0.

2I. Fernández M, Gavira L, Pérez M, Serrano R, Trujillo M. La sanidad desde el otro lado.Valoración social del sistema sanitario público en Andalucía. Sevilla: Consejería de Salud-Junta de Andalucía, 200 I.

22. Borrel C, García-Calvente MM, Martí-Boscá JV. La salud pública desde la perspectiva de género y clase social. Gac San 2004;18(I):2-6.

23. Delgado $A$. El papel del género en la relación médico-paciente. FMC 1999;6(8):509-16.

24. Delgado A, López-Fernández LA, Luna JD. Ser médico o médica marca diferencias en la práctica asistencial.Aten Prim 200I;28(4):219-226.

25. Riska E. Towards gender balance: but will women physicians have an impact on medicine? Soc Sci Med 200 I;52:179-187.

26. Ortiz-Gómez T, Birriel-Salcedo J, Ortega-del Olmo R. Género, profesiones sanitarias y salud pública. Gac San 2004;I8(Suppl I):I89-194. 27. Roter D, Lipkin M, Korsgaad A. Sex differences in patient's and physicians'comunication during primary care medical visits. Med Care 1991;29:1083-93.

28. Britt H, Bhasale A, Miles DA, Meza A, Sayer GP,Angelis M. The sex of the general practitioner.A comparison of characteristics, patient, and medical conditions manager. Med Care 1996;34:403-415.

29. Miqueo C, Tomás C, Tejero C, Barral MJ, Fernández T, Yayo T. Perspectivas de género en salud. Fundamentos científicos y socioprofesionales de diferencias sexuales no previstas. Madrid: Minerva, 200I. 